\title{
Hybrid Swarm Algorithms for Parameter Identification of an Actuator Model in an Electrical Machine
}

\author{
Ying Wu, ${ }^{1}$ Sami Kiviluoto, ${ }^{2}$ Kai Zenger, ${ }^{2}$ X. Z. Gao, ${ }^{2}$ and Xianlin Huang ${ }^{1}$ \\ ${ }^{1}$ Centre for Control Theory and Guidance Technology, Harbin Institute of Technology, Harbin 150000, China \\ ${ }^{2}$ Department of Automation and Systems Technology, Aalto University School of Electrical Engineering, P.O. Box 15500, \\ FI-00076 Aalto, Finland
}

Correspondence should be addressed to Kai Zenger, kai.zenger@tkk.fi

Received 15 December 2010; Accepted 4 March 2011

Academic Editor: Snehashish Chakraverty

Copyright () 2011 Ying Wu et al. This is an open access article distributed under the Creative Commons Attribution License, which permits unrestricted use, distribution, and reproduction in any medium, provided the original work is properly cited.

Efficient identification and control algorithms are needed, when active vibration suppression techniques are developed for industrial machines. In the paper a new actuator for reducing rotor vibrations in electrical machines is investigated. Model-based control is needed in designing the algorithm for voltage input, and therefore proper models for the actuator must be available. In addition to the traditional prediction error method a new knowledge-based Artificial Fish-Swarm optimization algorithm (AFA) with crossover, CAFAC, is proposed to identify the parameters in the new model. Then, in order to obtain a fast convergence of the algorithm in the case of a $30 \mathrm{~kW}$ two-pole squirrel cage induction motor, we combine the CAFAC and Particle Swarm Optimization (PSO) to identify parameters of the machine to construct a linear time-invariant(LTI) state-space model. Besides that, the prediction error method (PEM) is also employed to identify the induction motor to produce a black box model with correspondence to input-output measurements.

\section{Introduction}

One of the fundamental processes of control is the parameter identification. In control engineering a lot of effort has been done to develop methods to identify the system model and its parameters. A wide range of techniques such as the least squares method, the maximum likelihood method, and the cross correlation method, exist for system identification [1]. However, a drawback of traditional identification methods is their dependence on unrealistic assumptions such as unimodal performance landscapes and differentiability of the cost function. Consequently, some problems are oversimplified to fulfil such assumptions. Stochastic search algorithms such as evolutionary-based algorithms seem to be a promising alternative to these conventional methods. Swarm Intelligence is one of the most important branches for Evolutionary Algorithms, which has been widely used in identification problems [2-4].
The Artificial Fish-Swarm Algorithm (AFA) and the Particle Swarm Optimization (PSO) are two kinds of typical Swarm Intelligence methods [5]. The AFA is an animal behaviour-based optimization method and, like the PSO, which was firstly developed by $\mathrm{Li}$ and Eberhart and Kennedy, respectively [6-9]. PSO has been widely used in identification in many research fields [10-15]. However, there are few researches to apply the AFA in such kind of problems. The AFA can search for the global optimum effectively and has a certain adaptive ability for searching space. But the AFA individual behaviour is to hunt for local optimum. Therefore, avoiding individual premature becomes difficult. In this case, artificial fish will be stuck into local optima when dealing with multimodal optimization problems. To improve the performance of AFA is an im-portant challenge for applying the AFA in real problems [16].

In order to guide the evolutionary-based stochastic algorithms, a novel optimization method, the Cultural Algorithm 


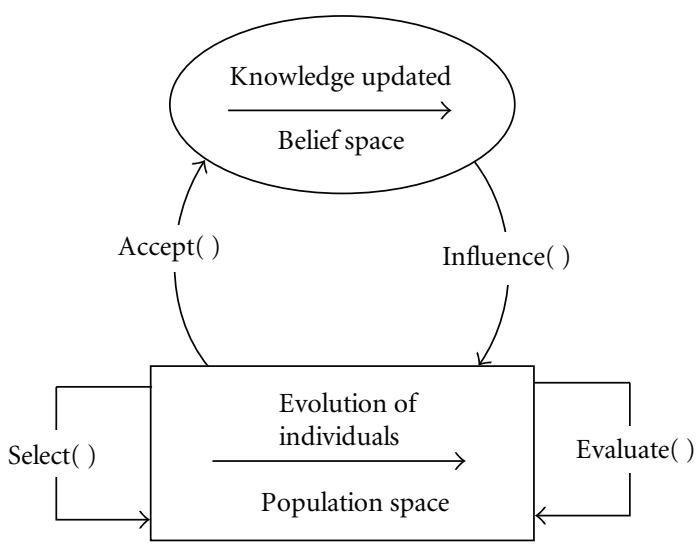

FIGURE 1: Culture algorithm framework.

(CA) proposed by Reynolds in 1994 was developed to demanding problems, due to its flexibility and efficiency [17]. The CA is a class of computational models derived from the principles of the culture evolution in nature, composed by the population space, the belief space and a communication protocol. In the CA, the evolution takes place in the population space. The knowledge generated from the experiences of the individuals is stored in the belief space. The communication protocol can be depicted as an acceptance function and an influence function, which is utilized to make interaction between the other two components [18, 19]. Various evolutionary algorithms have been utilized in the population space of the CA leading to good results [20-27]. The framework of the culture algorithm can be depicted in Figure 1.

The motivation behind this work is twofold. Firstly, in the literature there seems to be no results on hybrid AFA and CA. The knowledge stored in the belief space do act as a significant role in the process of evolution, and we try to find a proper framework for updating the belief space in combining the CA and AFA. Secondly, the new hybrid algorithm is applied to identify the parameters of a new kind of actuator, which is used to suppress rotor vibrations of an electronic machine.

As part of our investigation, two typical kinds of knowledge in CA, the situation knowledge and the normative knowledge, are stored in the belief space to update the population space and to establish the relationship between the two spaces in the CA. The performance of the CAFAC is explored using offline parameter identification of the actuator-rotor system in an electrical machine. The identification is performed based on a lower-order physical linear time invariant (LTI) parametric state-space model of the actuator-rotor system. The resulting model can be utilized to design model-based active control algorithms for vibrations reduction.

The rest of this paper is organized as follows. Section 2 briefly introduces the background knowledge of the system under control and the AFA. Section 3 proposes and discusses the underlying principle of the CAFAC. In Section 4 the identification of an actuator-rotor system is considered using
CAFAC and PEM and simulation results showing the control performance is established. A few conclusions and remarks are given in Section 5.

\section{Problem Statement and Preliminaries}

2.1. The Parametric Model of the Actuator-Rotor System. We examine a two-pole cage induction motor equipped with a built-in force actuator, which actively generates force on the rotor (Figure 2(a)). The actuator is a four-pole extra winding inside the stator slots (Figure 2(b)). This design allows producing additional forces without harming the normal operation of the motor. The actuator is controlled by two voltage signals that by design induce forces on the rotor in horizontal and vertical directions ( $x$ and $y)$.

The most important measurements for the identification are obtained using eddy current sensor. The sensors, conforming to the control signals, measure the rotor position also in horizontal and vertical directions. With them it is possible to record the rotor movement in any conditions accurately. Another set of sensors has been added on the right side of the motor in order to improve accuracy. Another important measurement devise is the encoder that provides the rotational angle and frequency of the rotor.

In the identification measurement the motor was operating at $32.085 \mathrm{~Hz}$ and the excitation input (control voltages in horizontal and vertical directions) was a uniform random number signal with frequency content up to $500 \mathrm{~Hz}$. The output data was then processed so that the effects of vibrations were removed and only the response to the excitation signals remained [28]. The processed measurement is displayed in Figure 3.

The data from this setup is used to obtain a mathematical actuator model that can be used for control design purposes, when the objective is compensating rotor vibrations. The motivation of obtaining a new parametric physical model is increased understanding of the model compared to a black box model, which has been used before successfully [29]. Parametric models have been identified before with data from a finite element model, but they do not describe the test motor and some of the identified parameters had complex values, although for a realistic machine the parameters such as resistances must be realvalued.

The linear time-invariant parametric (LTI) model of the system will be discussed. The model for the induction motor according to [30] is based on the mechanical model in modal coordinates [31]

$$
\begin{gathered}
\ddot{\eta}+2 \Omega \Xi \dot{\eta}+\Omega^{2}=\Phi_{r c}^{T} f_{c}+\Phi_{r c}^{T} f_{\mathrm{ex}}, \\
u_{r c}=\Phi_{r c} \eta,
\end{gathered}
$$

where $\eta$ is the modal coordinate vector, $u_{r c}$ is the displacement in $x$-direction and $y$-direction, $\Phi_{r c}$ is the modal matrix, $\Omega$ is a diagonal matrix, and $\Xi$ is the modal damping matrix, $f_{\text {ex }}$ denotes the sinusoidal disturbance causing rotor vibration disturbance, and $f_{c}$ the electro mechanical force 


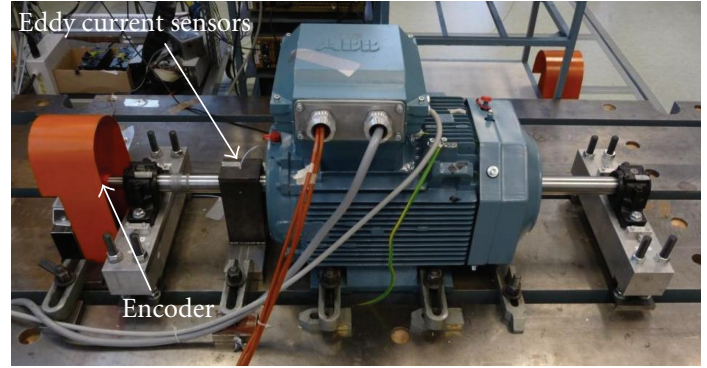

(a)

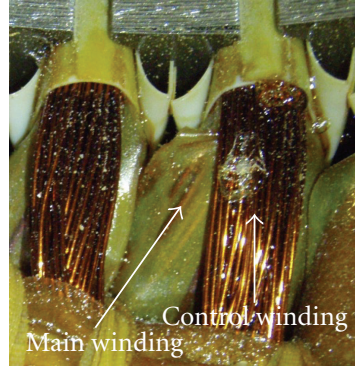

(b)

Figure 2: (a) The test motor [28]. Eddy current sensors are positioned on top of the rotor and behind it on the left. Encoder is located on the left side of the machine in the end of the rotor. (b) Control windings are built in the stator slots inside the motor [28].

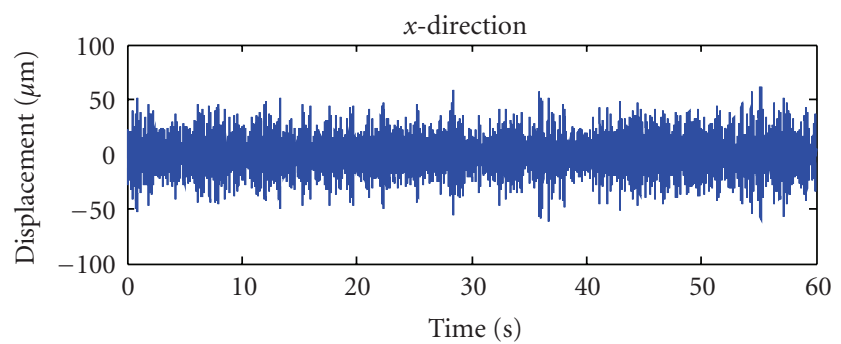

(a)

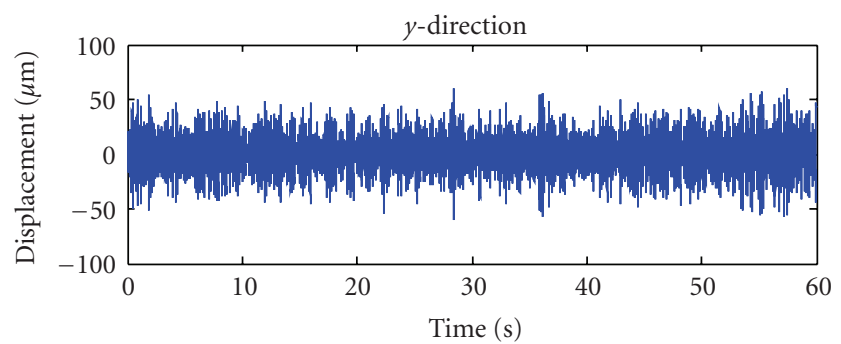

(b)

FIGURE 3: The response to control voltages in $x$ - and $y$-directions.

acting on the rotor. The model structure is the same as in [32]

$$
\begin{aligned}
\frac{d}{d t}\left(\begin{array}{l}
\dot{\eta} \\
\eta \\
i
\end{array}\right)= & {\left[\begin{array}{ccc}
-2 \Omega \Xi & \Phi_{r c}^{T} P_{e m} \Phi_{r c}-\Omega^{2} & \Phi_{r c}^{T} C_{e m} \\
I & 0 & 0 \\
S_{e m} \Phi_{r c} & Q_{e m} \Phi_{r c} & A_{e m}
\end{array}\right]\left(\begin{array}{l}
\dot{\eta} \\
\eta \\
i
\end{array}\right) } \\
& +\left[\begin{array}{cc}
0 & \Phi_{r c}^{T} \\
0 & 0 \\
B_{e m} & 0
\end{array}\right]\left(\begin{array}{c}
v \\
f_{\mathrm{ex}}
\end{array}\right), \\
u_{r c}= & {\left[\begin{array}{lll}
0 & \Phi_{r c} & 0
\end{array}\right]\left(\begin{array}{l}
\dot{\eta} \\
\eta \\
i
\end{array}\right) . }
\end{aligned}
$$

The input $v=\left(\operatorname{Re}\left(\underline{\hat{u}}_{c, 0}\right), \operatorname{Im}\left(\underline{\hat{u}}_{c, 0}\right)\right)$, is the control voltage, the first block row is the mechanical model, and $i=$ $\left(\operatorname{Re}\left(\hat{i}_{c, 0}\right), \operatorname{Re}\left(M_{r, c, 2} \hat{i}_{r, 2,0}\right)^{\mathrm{T}}, \operatorname{Im}\left(\hat{\underline{i}}_{c, 0}\right), \operatorname{Im}\left(M_{r, c, 2} \hat{\underline{i}}_{r, 2,0}\right)\right)^{\mathrm{T}}$, where $\hat{\underline{i}}_{c, 0}$ and $\hat{\underline{i}}_{r, 2,0}$ are current space vectors and $M_{r, c, 2}$ included in $i$ is mutual inductance of control winding and the rotor cage. In variables the subscript $c$ denotes a relation to the control winding, the subscript $r$ denotes a relation to the rotor cage inside the motor, and the number in subscript denotes harmonic component [33]. All the parameters for the model are based on measured data and their explanations are listed in Table 1 [30].
Following [30, 32] a combined electromechanical model can be derived starting from the voltage-flux equation for the control winding and the equation for rotor cage four-pole harmonic in stator coordinates

$$
\begin{aligned}
\hat{\underline{u}}_{c} & =R_{c} \hat{\hat{i}}_{c}+\frac{d \hat{\underline{\psi}}_{c, 2}}{d t}, \\
0 & =R_{r, 2} \hat{\hat{i}}_{r, 2}+\frac{d \underline{\hat{\psi}}_{r, 2}}{d t}-j 2 \omega_{m} \underline{\hat{\psi}}_{r, 2} .
\end{aligned}
$$

The four-pole harmonic flux leakages are

$$
\begin{aligned}
& \underline{\hat{\psi}}_{c, 2}=L_{c} \hat{\hat{i}}_{c}+M_{r, c, 2} \underline{\hat{i}}_{r, 2}+X_{c, \epsilon, 2} \underline{\hat{B}}_{1} \underline{z}_{r}, \\
& \underline{\hat{\psi}}_{r, 2}=L_{r, 2} \underline{\hat{i}}_{r, 2}+M_{c, r, 2} \underline{\hat{i}}_{c}+X_{r, \epsilon, 2} \underline{\hat{B}}_{1} \underline{z}_{r} .
\end{aligned}
$$

By substitution

$$
\begin{aligned}
\underline{\hat{\underline{u}}}_{c, 0}= & \left(R_{c}+j \omega_{1} L_{c}\right) \hat{\underline{i}}_{c, 0}+L_{c} \frac{d \hat{\underline{i}}_{c, 0}}{d t}+j \omega_{1} M_{r, c, 2} \hat{\underline{i}}_{r, 2,0} \\
& +\frac{d}{d t}\left(M_{r, c, 2} \hat{\underline{i}}_{r, 2,0}\right)+X_{c, \epsilon, 2} \underline{B}_{1}\left(\underline{\dot{z}}_{r}+j \omega_{1} \underline{z}_{r}\right),
\end{aligned}
$$


TABle 1: Parameters in the electromechanical model.

\begin{tabular}{|c|c|c|}
\hline Parameter & Unit & Explanation \\
\hline$R_{c}$ & $\mathrm{~m} \Omega$ & The resistance of the control winding. \\
\hline$L_{c}$ & $\mathrm{mH}$ & The inductance of the control winding. \\
\hline$k_{c} \underline{B}_{1}^{*}$ & $\mathrm{~N} /(\mathrm{A} \cdot \mathrm{m})$ & $\begin{array}{l}\text { The eccentricity coupling factor of the control winding multiplied with the complex conjugate of the first } \\
\text { air-gap field harmonic [34]. }\end{array}$ \\
\hline$M_{r, c, 2} \widetilde{M}_{c, r, 2}$ & $\mathrm{mH}$ & $\begin{array}{l}\text { Mutual inductance of rotor cage and control winding multiplied by itself and divided by rotor cage } \\
\text { inductance of four-pole harmonic }\left(M_{c, r, 2} \cdot M_{c, r, 2} / L_{r, 2}\right) \text {. }\end{array}$ \\
\hline$\gamma_{r, 2}$ & $1 / \mathrm{s}$ & $R_{r, 2} M_{c, r, 2} / L_{r, 2}$, where $R_{r, 2}$ is the rotor cage resistant of 4-pole harmonic. \\
\hline$X_{c, \epsilon, 2} \underline{B}_{1}$ & $\mathrm{~Wb} / \mathrm{m}$ & The eccentricity coupling factor of control winding multiplied with first air-gap field harmonic. \\
\hline$\alpha$ & $\mathrm{T}^{2}$ & $\begin{array}{l}\text { A coefficient related to the unbalanced magnetic pull towards the shortest air-gap (gap between stator and } \\
\text { rotor) [33]. }\end{array}$ \\
\hline$k_{r, 2} B_{1}^{*} / M_{r, c, 2}$ & $\mathrm{~N} /(\mathrm{m} \cdot \mathrm{Wb})$ & $\begin{array}{l}\text { The coupling factor of rotor cage multiplied with the complex conjugate of the first air-gap field harmonic } \\
\text { and divided by rotor cage inductance of four-pole harmonic. }\end{array}$ \\
\hline$\tilde{X}_{r, \epsilon, 2} \underline{B}_{1} / M_{r, c, 2}$ & $\mathrm{~Wb} / \mathrm{m}$ & $\begin{array}{l}\text { The eccentricity coupling factor of rotor cage four-pole harmonic multiplied with the first air-gap field } \\
\text { harmonic and divided by rotor cage inductance of four-pole harmonic. }\end{array}$ \\
\hline
\end{tabular}

where $\underline{z}$ is a complex coordinate representation of the rotor eccentricity. In addition, the control force $f_{c}$ in terms of the parameters in Table 1 is given by

$$
\begin{aligned}
f_{c}= & \underbrace{\frac{\pi d_{r} l_{r}}{4 \mu_{0} \delta_{0}}\left[\alpha \underline{z}_{r}+\underline{\beta} \underline{e}^{j 2 \omega_{1} t} \underline{z}_{r}^{*}\right]}_{\Rightarrow P_{e m}(t)} \\
& +\underbrace{\frac{\pi d_{r} l_{r}}{4 \delta_{0}} \underline{B}_{1}^{*}\left[\left(\frac{k_{r, 2}}{M_{r, c, 2}}\right) M_{r, c, 2} \hat{\hat{i}}_{r, 2,0}+k_{c} \hat{i}_{c, 0}\right]}_{\Rightarrow C_{e m}} .
\end{aligned}
$$

These equations include all the parameters to identify. Since the complex coordinate system of $\underline{z}$ is decomposed in real and imaginary parts in the final model, matrices $P_{e m}(t)$ and $C_{e m}$, are given by

$$
\begin{aligned}
P_{e m}(t)=\frac{\pi d_{r} l_{r}}{4 \mu_{0} \delta_{0}}( & {\left[\begin{array}{cc}
\operatorname{Re}\{\alpha\} & -\operatorname{Im}\{\alpha\} \\
\operatorname{Im}\{\alpha\} & \operatorname{Re}\{\alpha\}
\end{array}\right] } \\
& \left.+\left[\begin{array}{cc}
\operatorname{Re}\left\{\beta e^{j 2 \omega_{1} t}\right\} & \operatorname{Im}\left\{\beta e^{j 2 \omega_{1} t}\right\} \\
\operatorname{Im}\left\{\beta e^{j 2 \omega_{1} t}\right\} & -\operatorname{Re}\left\{\beta e^{j 2 \omega_{1} t}\right\}
\end{array}\right]\right), \\
C_{e m C}= & \left.\frac{\pi d_{r} l_{r}\left[k_{c} \underline{B}_{1}^{*}\right.}{4 \delta_{0}}\left(\frac{k_{r, 2}}{M_{r, c, 2}}\right) \underline{B}_{1}^{*}\right], \\
C_{e m}= & {\left[\begin{array}{ll}
\operatorname{Re}\left\{C_{e m C}\right\} & -\operatorname{Im}\left\{C_{e m C}\right\} \\
\operatorname{Im}\left\{C_{e m C}\right\} & \operatorname{Re}\left\{C_{e m C}\right\}
\end{array}\right] . }
\end{aligned}
$$

It should be noted that in the time-invariant case $P_{e m}(t)$ is constant $P_{\text {em }}$.

Equation (6) can be written in matrix form

$$
\begin{aligned}
& {\left[\begin{array}{cr}
L_{c} & 1 \\
M_{r, c, 2} \widetilde{M}_{c, r, 2} & 1
\end{array}\right] \times \frac{d}{d t}\left[\begin{array}{c}
\hat{i}_{c, 0} \\
M_{r, c, 2} \hat{\hat{i}}_{r, 2,0}
\end{array}\right]} \\
& +\left[\begin{array}{cc}
R_{c}+j \omega_{1} L_{c} & j \omega_{1} \\
j \omega_{2} M_{r, c, 2} \widetilde{M}_{c, r, 2} & \gamma_{r, 2}+j \omega_{2}
\end{array}\right]\left[\begin{array}{c}
\hat{i}_{c, 0} \\
M_{r, c, 2} \hat{\hat{i}}_{r, 2,0}
\end{array}\right] \\
& +\left[\begin{array}{c}
X_{c, \epsilon, 2} \underline{B}_{1} \\
M_{r, c, 2} \tilde{X}_{r, \epsilon, 2} \underline{B}_{1}
\end{array}\right] \underline{\dot{z}}_{r}+\left[\begin{array}{c}
j \omega_{1} X_{c, \epsilon, 2} \underline{B}_{1} \\
j \omega_{1} M_{r, c, 2} \tilde{X}_{r, \epsilon, 2} \underline{B}_{1}
\end{array}\right] \underline{z}_{r}=\left[\begin{array}{c}
1 \\
0
\end{array}\right] \underline{\hat{u}}_{c, 0} .
\end{aligned}
$$

Rearranging this will give the rest of the matrices, which are needed for the combined model

$$
\begin{aligned}
& \frac{d}{d t}\left[\begin{array}{c}
\hat{\hat{i}}_{c, 0} \\
M_{r, c, 2} \underline{\hat{i}}_{r, 2,0}
\end{array}\right] \\
& =\underbrace{-\left[\begin{array}{cc}
L_{c} & 1 \\
M_{r, c, 2} \widetilde{M}_{c, r, 2} & 1
\end{array}\right]^{-1}\left[\begin{array}{c}
X_{c, \epsilon, 2} \underline{B}_{1} \\
M_{r, c, 2} \tilde{X}_{r, \epsilon, 2} \underline{\hat{B}}_{1}
\end{array}\right]}_{S_{e m C}} \dot{\underline{z}}_{r} \\
& \underbrace{-\left[\begin{array}{cc}
L_{c} & 1 \\
M_{r, c, 2} \widetilde{M}_{c, r, 2} & 1
\end{array}\right]^{-1}\left[\begin{array}{c}
j \omega_{1} X_{c, \epsilon, 2} \underline{B}_{1} \\
j \omega_{1} M_{r, c, 2} \tilde{X}_{r, \epsilon, 2} \underline{B}_{1}
\end{array}\right]}_{Q_{e m C}} \underline{z}_{r} \\
& \underbrace{-\left[\begin{array}{cr}
L_{c} & 1 \\
M_{r, c, 2} \widetilde{M}_{c, r, 2} & 1
\end{array}\right]^{-1}\left[\begin{array}{cc}
R_{c}+j \omega_{1} L_{c} & j \omega_{1} \\
j \omega_{2} M_{r, c, 2} \widetilde{M}_{c, r, 2} & \gamma_{r, 2}+j \omega_{2}
\end{array}\right]}_{A_{e m C}}
\end{aligned}
$$




$$
\times\left[\begin{array}{c}
\hat{\underline{\hat{i}}}_{c, 0} \\
M_{r, c, 2} \hat{\underline{i}}_{r, 2,0}
\end{array}\right]+\underbrace{\left[\begin{array}{cc}
L_{c} & 1 \\
M_{r, c, 2} \widetilde{M}_{c, r, 2} & 1
\end{array}\right]^{-1}\left[\begin{array}{l}
1 \\
0
\end{array}\right]}_{B_{e m C}} \hat{\underline{\underline{u}}}_{c, 0} .
$$

Matrices $A_{e m}, B_{e m}, S_{e m}$, and $Q_{e m}$ are finally obtained like $C_{e m}$ in (10)

$$
\begin{aligned}
A_{e m} & =\left[\begin{array}{cc}
\operatorname{Re}\left\{A_{e m C}\right\} & -\operatorname{Im}\left\{A_{e m C}\right\} \\
\operatorname{Im}\left\{A_{e m C}\right\} & \operatorname{Re}\left\{A_{e m C}\right\}
\end{array}\right], \\
B_{e m} & =\left[\begin{array}{cc}
\operatorname{Re}\left\{B_{e m C}\right\} & -\operatorname{Im}\left\{B_{e m C}\right\} \\
\operatorname{Im}\left\{B_{e m C}\right\} & \operatorname{Re}\left\{B_{e m C}\right\}
\end{array}\right], \\
S_{e m} & =\left[\begin{array}{cc}
\operatorname{Re}\left\{S_{e m C}\right\} & -\operatorname{Im}\left\{S_{e m C}\right\} \\
\operatorname{Im}\left\{S_{e m C}\right\} & \operatorname{Re}\left\{S_{e m C}\right\}
\end{array}\right], \\
Q_{e m} & =\left[\begin{array}{cc}
\operatorname{Re}\left\{Q_{e m C}\right\} & -\operatorname{Im}\left\{Q_{e m C}\right\} \\
\operatorname{Im}\left\{S_{e m C}\right\} & \operatorname{Re}\left\{Q_{e m C}\right\}
\end{array}\right] .
\end{aligned}
$$

These matrices and the mechanical model (1) can be combined in one state-space model (3).

The parameters of the electromechanical model are listed in Table 1. Certain practical constraints were set for the parameters for fulfilling physical quantities. Here the resistance is constant and known (14.5), so it does not need to be optimized. When the effect of the unbalanced magnetic pull is ignored we can get the LTI model. The input $u(t)$ consists of the voltage input $v$ to the control winding and the disturand the outputs are the displacements $u_{r c}$ of the rotor center. The system matrices $A, B$, and $C$ are the functions of the unknown parameters in the model which are reformed in a vector as

$$
P=\left\{L_{c}, k_{c} \underline{B}_{1}^{*}, M_{r, c, 2} \widetilde{M}_{c, r, 2}, \gamma_{r, 2}, X_{c, \epsilon, 2} \underline{B}_{1}, \alpha, \frac{k_{r, 2} \underline{B}_{1}^{*}}{M_{r, c, 2}}, \frac{\tilde{X}_{c, \epsilon, 2} \underline{B}_{1}}{M_{r, c, 2}}\right\} .
$$

The corresponding significances of the unknown parameters, and the detailed derivation of the model can be found in [32].

2.2. The Artificial Fish-Swarm Algorithm. Suppose that the problem under consideration has $D$-dimensions. Initialize the swarm with $N$ artificial fish. The state of one artificial fish can be formulated as $X_{i}=\left(x_{i 1}, x_{i 2}, \ldots, x_{i D}\right), i=$ $1, \ldots, N$, where $X_{i}$ represents the target variable for the problem under consideration. $y=f\left(X_{i}\right)$ stands for the food concentration of the artificial fish currently, where it is the objective function. The basic behaviours of artificial fish can be expressed as follows $\left(d_{i j}=\left\|X_{j}-X_{i}\right\|\right.$ stands for the Euclidean distance between $X_{i}$ and $X_{j}$, "vd" is visual distance of the artificial fish individual, " $s$ " is the size of the movement of artificial fish, $\delta$ is the crowd factor of the artificial fish) $[6]$.
(1) Preying. The current state of the artificial fish is $X_{i}$, the artificial fish selects a state $X_{j}$ randomly within the visual distance, such as $X_{j}=X_{i}+\operatorname{rand}(0,1) \times$ vd. If $f\left(X_{j}\right)<f\left(X_{i}\right)$ the artificial fish moves from $X_{i}$ towards $X_{j}$, meaning that $X_{i}^{t} \rightarrow X_{i}^{t+1}$. The formulation can be depicted as follows:

$$
X_{i}^{t+1}=X_{i}^{t}+\operatorname{rand}(0,1) \times s \times \frac{X_{j}^{t}-X_{i}^{t}}{\left\|X_{j}^{t}-X_{i}^{t}\right\|} .
$$

If $f\left(X_{j}\right)>f\left(X_{i}\right)$, the artificial fish selects another state randomly again. If the artificial fish cannot meet the requirement in a given time, then it moves one step randomly as

$$
X_{i}^{t+1}=X_{i}^{t}+\operatorname{rand}(0,1) \times s
$$

(2) Swarming. The current state of the artificial fish is $X_{i}, \mathrm{nf}$ is the number of its fellows within the visual distance, equal to the number of elements in the set of $B=\left\{X_{j} \mid d_{i j} \leq\right.$ Visual $\}$. If $\mathrm{nf} \neq 0$ that is the set $B$ is not empty, let $X_{\text {center }}=$ $\sum_{j=1}^{\mathrm{nf}} X_{j} / \mathrm{nf}$ and then $y_{\text {center }}=f\left(X_{\text {center }}\right)$ stands for the fitness of the centre position. If $\mathrm{nf} \times y_{\text {center }}<\delta \times y_{i}$, meaning that this area is not crowded, then if $y_{\text {center }}<y_{i}$, the artificial fish moves one step towards the centre position:

$$
X_{i}^{t+1}=X_{i}^{t}+\operatorname{rand}(0,1) \times s \times \frac{X_{c}^{t}-X_{i}^{t}}{\left\|X_{c}^{t}-X_{i}^{t}\right\|} .
$$

Otherwise it executes the behaviour of preying.

(3) Chasing. The current state of the artificial fish is $X_{i}$, $X_{\min }$ stands for the best artificial fish individual within $X_{i}$ 's visual distance. $\mathrm{nf}$ is the number of $X_{\min }$ 's fellows within the visual distance. $y_{\min }=f\left(X_{\min }\right)$, if $y_{\min }<y_{i}$ and $\mathrm{nf} \times y_{\min }<$ $\delta \times y_{i}$, the artificial fish moves one step to $X_{\min }$ :

$$
X_{i}^{t+1}=X_{i}^{t}+\operatorname{rand}(0,1) \times s \times \frac{X_{\min }^{t}-X_{i}^{t}}{\left\|X_{\min }^{t}-X_{i}^{t}\right\|} .
$$

Otherwise it executes the behaviour of preying.

\section{Hybrid Optimization Methods of Articificial Fish Algorithm and Culture Algorithm}

Here, the fish swarm is regarded as the population space, where the domain knowledge is extracted from. Then the domain knowledge is formed and stored in belief space so as to model and impact the evolution of the population at iteration. In four versions of CAFAC, we use the situation knowledge and the normative knowledge to guide the direction and the step size of the evolution. Both of them can be depicted as follows.

3.1. Structures of Belief Space in CAFAC. The situational knowledge provides a set of best individuals available for the interpretation of specific individual experience [19]. Here, the situational exemplar set consists of only the best fish found so far, $S=\left\langle S^{t} \mid S^{t}=\left\{s_{1}^{t}, s_{2}^{t}, \ldots, s_{n}^{t}\right\}\right\rangle, S^{t}$ stands for 
the best fish in the swarm at iteration $t$. In other words, it can be initialized with the best fish in the initial fish swarm and updated by the following function:

$$
s_{j}^{t+1}= \begin{cases}X_{g \text { best }, j}^{t+1} & \text { if } f\left(X_{\text {gbest }}^{t+1}\right)<f\left(s^{t}\right), \\ s_{j}^{t} & \text { otherwise }\end{cases}
$$

where $X_{\text {gbest }}^{t+1}$ denotes the best artificial fish individual in the fish swarm at generation $t+1$.

The normative knowledge can give the feasible solution space of the optimization problems under consideration [19]. It is a set of information for each variable and is given as

$$
N=\langle I, U, L, D\rangle
$$

where $U, L$ and $D$ are $n$-dimensional vectors, and $I=$ $\{x \mid l \leq x \leq u\}, n$ is the number of the variables, $l_{j}$ and $u_{j}$ are the lower and upper bounds for the $j$ th variable, respectively, $L_{j}$ and $U_{j}$ are the values of the fitness function associated with the bound $l_{j}$ and $u_{j}$. Generally, $l_{j}$ and $u_{j}$ are initialized with the lower and upper bounds of individuals. $L_{j}$ and $U_{j}$ are usually initialized with positive infinity. The formulation for normative knowledge updating can be depicted in Table 2.

3.2. Acceptance Function in CAFAC. The acceptance function determines which individuals and their performances can have impact on the knowledge in the belief space. The number of the individuals accepted for the update of the belief space is obtained according to the following function [1]:

$$
f_{a}(N, t)=N \cdot \beta+\left\lfloor N \cdot \frac{\beta}{t}\right\rfloor,
$$

where $N$ is the size of the swarm, $t$ is the iteration number, and $\beta$ is a constant (usually chosen as 0.2 ).

3.3. Influence Functions in CAFAC. The belief space can influence the evolution in the population space in three ways:

(i) determining the step size of the evolution,

(ii) determining the direction of the evolution,

(iii) determining the visual distance of AFA.

More precisely, if the normative knowledge is used to determine the step size of the evolution and visual distance in AFA, our knowledge-based AFA is named as CAFAC (Ns). In four versions of CAFAC, all of the behaviours, preying, swarming, and chasing, are modelled by the knowledge. The influence function for the CAFAC is defined as in Tables 3, 4 and 5 .

If the situational knowledge is used to guide the direction of the evolution, our knowledge-based AFA is named as CAFAC (Sd).

If the normative knowledge guides the step size and the visual distance meanwhile the situational knowledge is used to determine the direction of the evolution, respectively, our knowledge-based AFA is named as CAFAC (Ns+Sd).

If the normative knowledge is used to determine the step size and direction of the evolution and the visual distance, our knowledge-based AFA is named as CAFAC (Ns+Nd).

In Tables 3, 4, and 5, $\operatorname{size}\left(I_{k}\right)=u_{k}-l_{k}$ is the size of the belief interval which is decided by the normative knowledge for the $k$ th variable. The $\operatorname{rand}(0,1)$ is a random number uniformly distributed in the interval $(0,1)$. The other parameters are depicted in Section 2.2.

3.4. Crossover Operator. A criterion is set up to judge whether the algorithm falls into local optimum:

$$
\left|\frac{f\left(X_{i}^{t}\right)-f\left(X_{i}^{t-1}\right)}{f\left(X_{i}^{t-1}\right)}\right|<0.1 .
$$

When the criterion is satisfied, the crossover operator will be applied to the $i$ th artificial fish $X_{i}(i=1, \ldots, N)$ :

$$
x_{i}^{\prime}=x_{r 1}+\alpha \times\left(x_{r 2}-x_{r 1}\right),
$$

where $x_{r 2}, x_{r 1}$ are two individuals selected randomly, $r_{1}, r_{2}$ are integers, which are generated randomly in the interval of $[1, N]$ and $r_{1} \neq r_{2} \neq i . \alpha$ is random number uniformly distributed in interval of $[-d, 1+d]$ and $d$ is constant $(0.25)$. Evaluate the child $x_{i}^{\prime}$ and replace the individual $x_{i}$ with the child if $x_{i}^{\prime}$ performs better [35].

\section{Application for Parameter Identification of Induction Motor}

4.1. Identification Using CAFAC and PEM. In the identification process we disturb the system by voltage $v$ and force $f_{\text {ex }}$ excitations. In order to decrease the dimension of the problem we remove the disturbance from the data. So the input $f_{\text {ex }}$ is set to zero. Then the problem to be considered is to match the model output with processed data using the recorded band limited white noise signal as input $v$. The basic idea of parameter estimation is to find a vector $P$ to minimize the following cost function:

$$
J(P)=\frac{\sum_{n=1}^{N}\left|\hat{u}_{r c}(n)-u_{r c}(n)\right|}{N},
$$

where $u_{r c}(n)$ are the measurement of output for the real system under certain inputs; $\hat{u}_{r c}(n)$ is the estimated output by exciting the model (1)-(2) using the same inputs. The experiments are carried out using a simulated reference signal generated by the real system's parameters, which have been verified by simulations for a small $30 \mathrm{~kW}$ two-pole cage induction motor.

Here we use an indicator of fit value to evaluate the accuracy of an identified model for a specific parameters vector $P$ and given inputs $v(t)$ and $f_{\mathrm{ex}}(t)$. The fit value is computed as [30]

$$
m d=100 \times\left(1-\frac{J(P)}{\left(\sum_{n=1}^{N}\left|\hat{u}_{r c}(n)-\bar{u}_{r c}\right|^{2}\right)^{1 / 2}}\right)
$$


TABLE 2: Formulation for normative knowledge updating.

$l_{j}^{t+1}=\left\{\begin{array}{ll}x_{i, j} & \text { if } x_{i, j} \leq l_{j}^{t} \text { or } f\left(X_{i}\right)<L_{j}^{t} \\ l_{j}^{t}, & \text { otherwise }\end{array} \quad L_{j}^{t+1}= \begin{cases}f\left(X_{i}\right) & \text { if } x_{i, j} \leq l_{j}^{t} \text { or } f\left(X_{i}\right)<L_{j}^{t} \\ L_{j}^{t}, & \text { otherwise }\end{cases} \right.$
$u_{j}^{t+1}=\left\{\begin{array}{ll}x_{k, j} & \text { if } x_{k, j} \geq u_{j}^{t} \text { or } f\left(X_{k}\right)<U_{j}^{t} \\ u_{j}^{t}, & \text { otherwise }\end{array} \quad U_{j}^{t+1}= \begin{cases}f\left(X_{k}\right) & \text { if } x_{k, j} \geq u_{j}^{t} \text { or } f\left(X_{k}\right)<U_{j}^{t} \\ U_{j}^{t}, & \text { otherwise }\end{cases} \right.$
where the $i$ th individual affects the lower bound for variable $j$, and the $k$ th individual affects the upper bound for variable $j$.
Note that $t$ denotes the current generation of the belief space.

TABLE 3: Influence function for swarming.

\begin{tabular}{|c|c|c|c|c|}
\hline \multicolumn{5}{|c|}{ Swarming } \\
\hline Ns & \multicolumn{4}{|c|}{$x_{i k}^{t+1}=x_{i k}^{t}+\frac{\operatorname{size}\left(I_{k}\right) \times \operatorname{rand}(0,1) \times\left(x_{c k}^{t}-x_{i k}^{t}\right)}{\left\|X_{c}^{t}-X_{i}^{t}\right\|}$} \\
\hline Sd & $x_{i k}^{t+1}=$ & $\begin{array}{l}x_{i k}^{t}+ \\
x_{i k}^{t}- \\
x_{i k}^{t}+\end{array}$ & $\begin{array}{l}\frac{\operatorname{rand}(0,1) \times s \times\left(x_{c k}^{t}-x_{i k}^{t}\right)}{\left\|X_{c}^{t}-X_{i}^{t}\right\|} \\
\frac{\operatorname{rand}(0,1) \times s \times\left(x_{c k}^{t}-x_{i k}^{t}\right)}{\left\|X_{c}^{t}-X_{i}^{t}\right\|} \\
\frac{\operatorname{and}(0,1) \times s \times\left(x_{c k}^{t}-x_{i k}^{t}\right)}{\left\|X_{c}^{t}-X_{i}^{t}\right\|}\end{array}$ & $\begin{array}{l}<s_{k}^{t} \\
>s_{k}^{t} \\
\text { wise }\end{array}$ \\
\hline NsSd & $x_{i k}^{t+1}=$ & $\begin{array}{l}x_{i k}^{t}+ \\
x_{i k}^{t}- \\
x_{i k}^{t}+\end{array}$ & $\begin{array}{l}\frac{\operatorname{size}\left(I_{k}\right) \times \operatorname{rand}(0,1) \times\left(x_{c k}^{t}\right.}{\left\|X_{c}^{t}-X_{i}^{t}\right\|} \\
\frac{\operatorname{size}\left(I_{k}\right) \times \operatorname{rand}(0,1) \times\left(x_{c k}^{t}\right.}{\left\|X_{c}^{t}-X_{i}^{t}\right\|} \\
\frac{\operatorname{ize}\left(I_{k}\right) \times \operatorname{rand}(0,1) \times\left(x_{c k}^{t}-\right.}{\left\|X_{c}^{t}-X_{i}^{t}\right\|}\end{array}$ & $\begin{array}{l}\text { if } x_{i k}^{t}<s_{k}^{t} \\
\text { if } x_{i k}^{t}>s_{k}^{t} \\
\text { otherwise }\end{array}$ \\
\hline $\mathrm{NsNd}$ & $x_{i k}^{t+1}=\{$ & $\begin{array}{l}x_{i k}^{t}+ \\
x_{i k}^{t}- \\
x_{i k}^{t}+\end{array}$ & $\begin{array}{l}\frac{\operatorname{size}\left(I_{k}\right) \times \operatorname{rand}(0,1) \times\left(x_{c k}^{t}\right.}{\left\|X_{c}^{t}-X_{i}^{t}\right\|} \\
\frac{\operatorname{size}\left(I_{k}\right) \times \operatorname{rand}(0,1) \times\left(x_{c k}^{t}\right.}{\left\|X_{c}^{t}-X_{i}^{t}\right\|} \\
\frac{\operatorname{ize}\left(I_{k}\right) \times \operatorname{rand}(0,1) \times\left(x_{c k}^{t}-\right.}{\left\|X_{c}^{t}-X_{i}^{t}\right\|}\end{array}$ & $\begin{array}{l}\text { if } x_{i k}^{t}<l_{k}^{t} \\
\text { if } x_{i k}^{t}>u_{k}^{t} \\
\text { otherwise }\end{array}$ \\
\hline
\end{tabular}

where $\bar{u}_{r c}$ is the average value of the measurement data $\left\{u_{r c}(n)\right\}$. This indicator was chosen because it has been used in the research of optimal control of an induction motor using the prediction error method (PEM) for identification.

In our previous work, we found that the NsSd version has better performance than the three others we mentioned in Section 3 [26]. Even if the CAFAC has better optimization function than PSO, the speed of the PSO is an obvious advantage. Therefore we combine the CAFAC and the basic PSO so as to speed up the optimization process. The optimization process is implemented using the software MATAB. The basic procedure of CAFAC algorithm can be described as follows.

(1) Set all the values for the parameters and initialize the $N$ artificial fish in the search scope with random positions.

(2) Evaluate all the artificial fishes using the fitness function $y$ and initialize the belief space.
(3) For each ith artificial fish, simulate the preying pattern, swarming, and chasing patterns separately, and select the best child fish. If the child is better, replace the $i$ th artificial fish with the child.

(4) Update the belief space.

(5) If the crossover criterion is satisfied, apply the crossover operator to the $i$ th artificial fish got from Step (3).

(6) Switch to PSO until the termination criterion is satisfied.

(7) Switch to (3) until the termination criterion is satisfied.

(8) End the program if the final termination criterion is satisfied.

The termination criterion here is the same as (22) and the final termination criterion is the maximum of the whole iteration. 
TABLE 4: Influence function for preying.

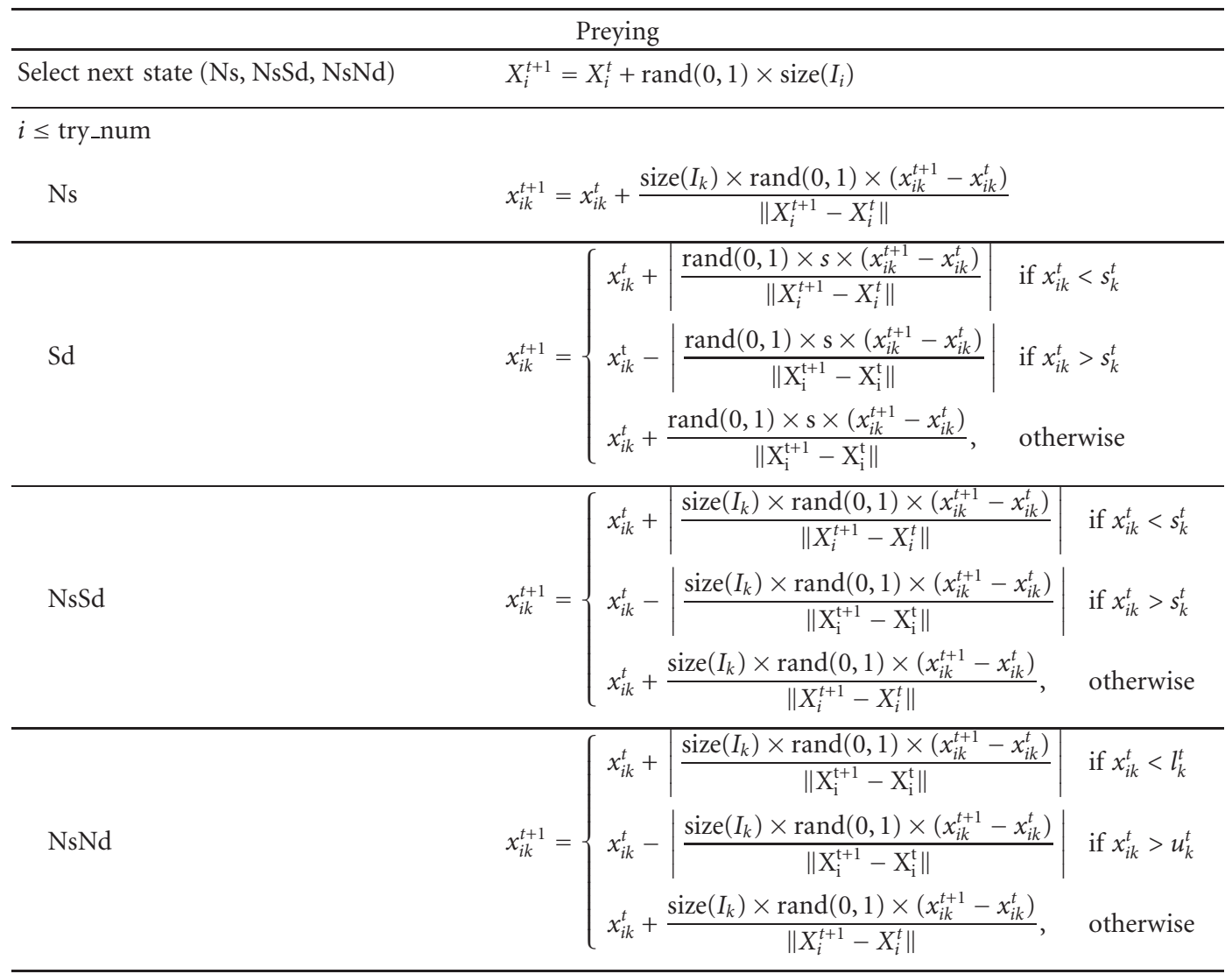

$i>$ try_num

Ns $\quad x_{i k}^{t+1}=x_{i k}^{t}+\operatorname{size}\left(I_{k}\right) \times \operatorname{rand}(0,1)$

\begin{tabular}{|c|c|c|}
\hline $\mathrm{Sd}$ & $x_{i k}^{t+1}=$ & $\begin{array}{ll}x_{i k}^{t}+|\operatorname{rand}(0,1) \times s| & \text { if } x_{i k}^{t}<s_{k}^{t} \\
x_{i k}^{t}-|\operatorname{rand}(0,1) \times s| & \text { if } x_{i k}^{t}>s_{k}^{t} \\
x_{i k}^{t}+\operatorname{rand}(0,1) \times s, & \text { otherwise }\end{array}$ \\
\hline NsSd & $x_{i k}^{t+1}=$ & $\begin{array}{ll}x_{i k}^{t}+\left|\operatorname{size}\left(I_{k}\right) \times \operatorname{rand}(0,1)\right| & \text { if } x_{i k}^{t}<s_{k}^{t} \\
x_{i k}^{t}-\left|\operatorname{size}\left(I_{k}\right) \times \operatorname{rand}(0,1)\right| & \text { if } x_{i k}^{t}>s_{k}^{t} \\
x_{i k}^{t}+\operatorname{size}\left(I_{k}\right) \times \operatorname{rand}(0,1), & \text { otherwise }\end{array}$ \\
\hline NsNd & $x_{i k}^{t+1}=$ & $\begin{array}{ll}x_{i k}^{t}+\left|\operatorname{size}\left(I_{k}\right) \times \operatorname{rand}(0,1)\right| & \text { if } x_{i k}^{t}<l_{k}^{t} \\
x_{i k}^{t}-\left|\operatorname{size}\left(I_{k}\right) \times \operatorname{rand}(0,1)\right| & \text { if } x_{i k}^{t}>u_{k}^{t} \\
x_{i k}^{t}+\operatorname{size}\left(I_{k}\right) \times \operatorname{rand}(0,1), & \text { otherwise }\end{array}$ \\
\hline
\end{tabular}

In PEM identification the general idea is to produce a model that minimizes a norm, such as

$$
V_{N}\left(\theta, Z^{N}\right)=\frac{1}{N} \sum_{t=0}^{N} \ell\left(\varepsilon_{F}(t, \theta)\right),
$$

where $\varepsilon_{F}(t, \theta)$ is the prediction error filtered through a linear filter and $\ell(\cdot)$ is a scalar function [36]. $Z^{N}$ is the data consisting of $N$ samples and $\theta$ is the model parameterization (i.e., polynomials). The problem is usually solved iteratively using gradient-based methods.

With the identification data, the PEM identification will result in fit values of 79.1 and 80.6 in horizontal and vertical directions. The CAFAC identification produced a model that had fit values of 73.9 and 77.7. Figure 4 displays the filtered measurement, and the model outputs from PEM and the parametric model. It can be stated that the models estimate the data reasonably well. The main differences occur near zero where the response is modest.

4.2. Simulation Results. The obtained model was tested in simulations by implementing a state observer and a linear quadratic controller (LQ) for the model. The same was done in [29] with a model, which was identified using PEM. A real measurement of rotor vibrations (similar to 
TABLE 5: Influence function for chasing.

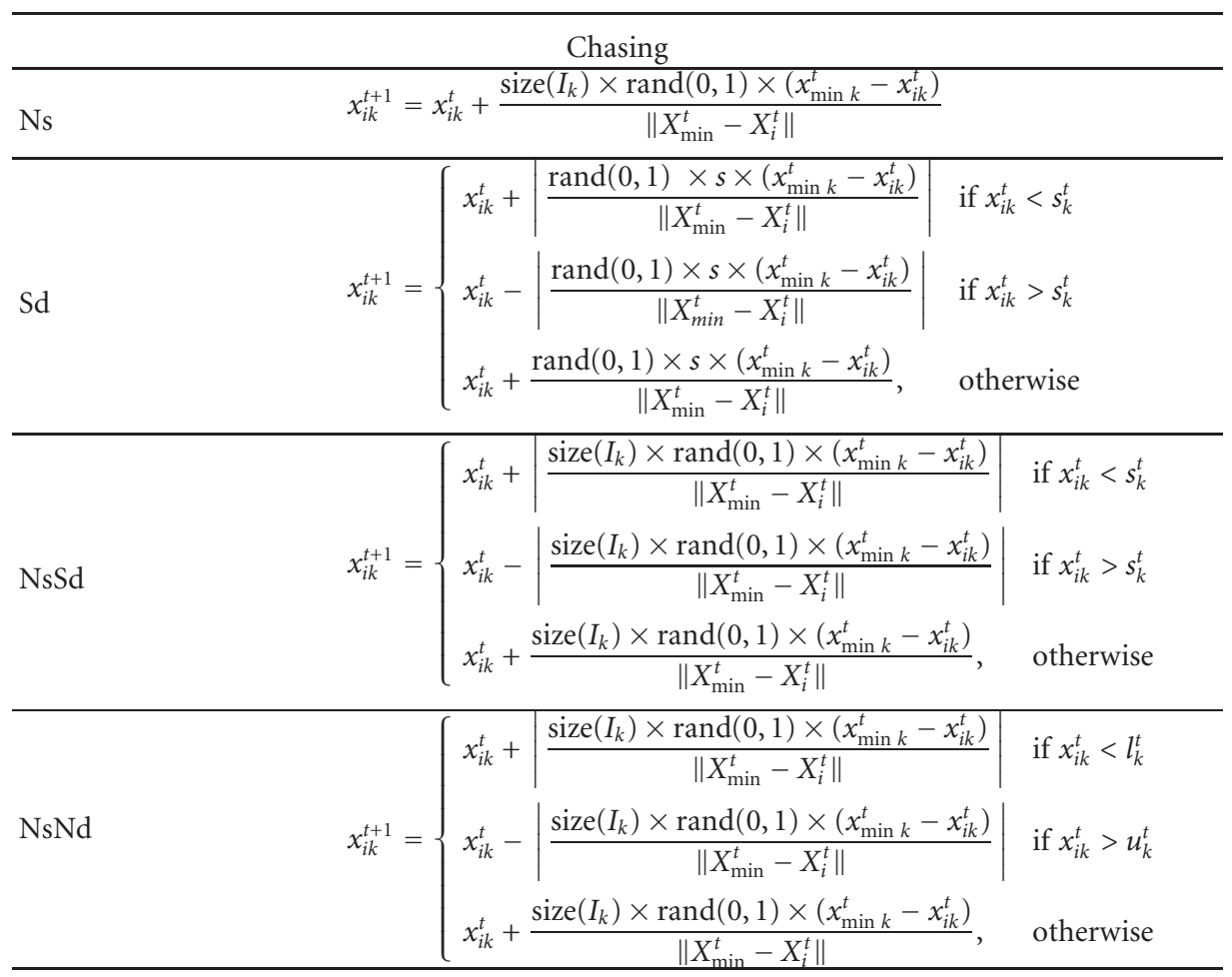

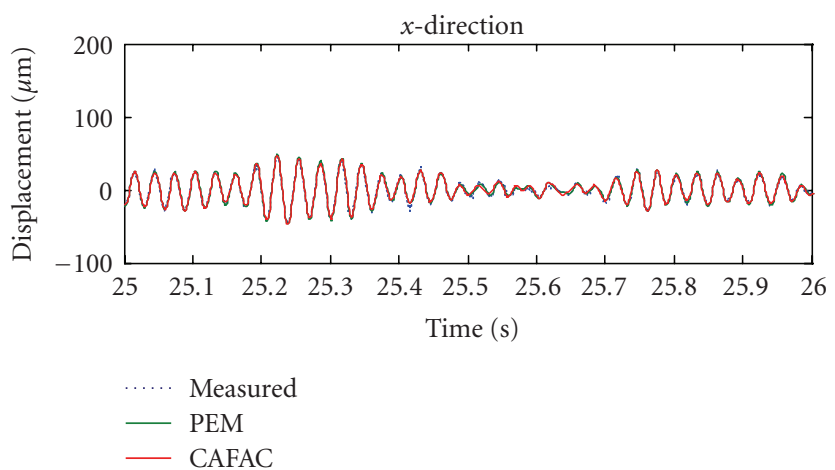

(a)

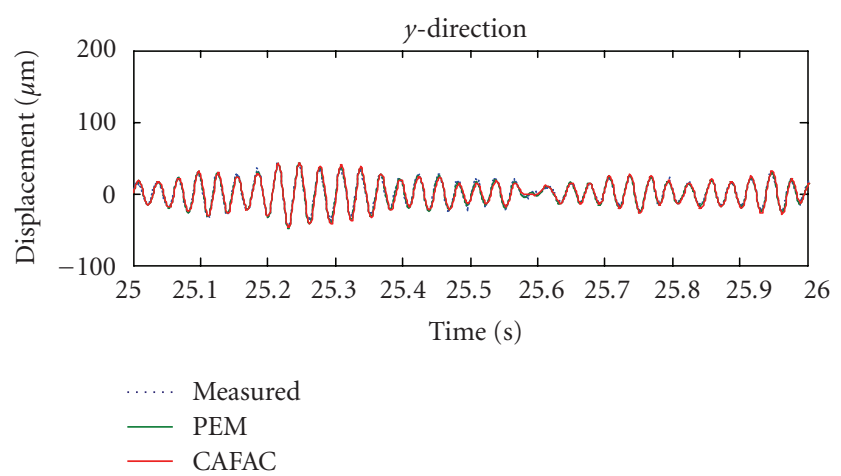

(b)

Figure 4: Measured data and modelled outputs using PEM and CAFAC identification methods.

the identification data, but including the disturbance and not input signal) in the 2-pole induction motor was used as the input in the simulations. The expected result was that the observer would follow this signal and the LQ controller, fed through the model of the actuator and would compensate the vibrations. Figure 5 shows the estimation error of the observer. It can be concluded that the observer works decently since the amplitude of the estimation error is about $5 \mu \mathrm{m}$ and the measured amplitude varies between 130 and $240 \mu \mathrm{m}$. The control result is displayed in Figure 6, where the controller is switched on after three seconds. During the 17 -second period when control is on, the reductions of the vibrations are up to $67.0 \%$ and $70.3 \%$ and the control also reduces the fluctuation of the amplitude, which depends on the slip angle.
It is also necessary to consider the control signal because the test equipment can produce voltages in the range of $\pm 100 \mathrm{~V}$, and naturally the voltage levels should not be very high in a properly working controller. Figure 7 shows that there is at first a peak in the control signal, which reaches the saturation level, but it settles close to $\pm 20 \mathrm{~V}$ range after 0.02 seconds.

The same simulations were also done with the black box model, which was identified using PEM, and the controller was tuned in the same manner. In this case the controller reduced vibrations in the simulations by $70.0 \%$ and $64.2 \%$. The result is displayed in Figure 8. The control signals with the PEM model stay between $\pm 50 \mathrm{~V}$ after that (see Figure 9). Strictly based on this data, the parametric model is slightly better than the PEM identification. However, the parametric 


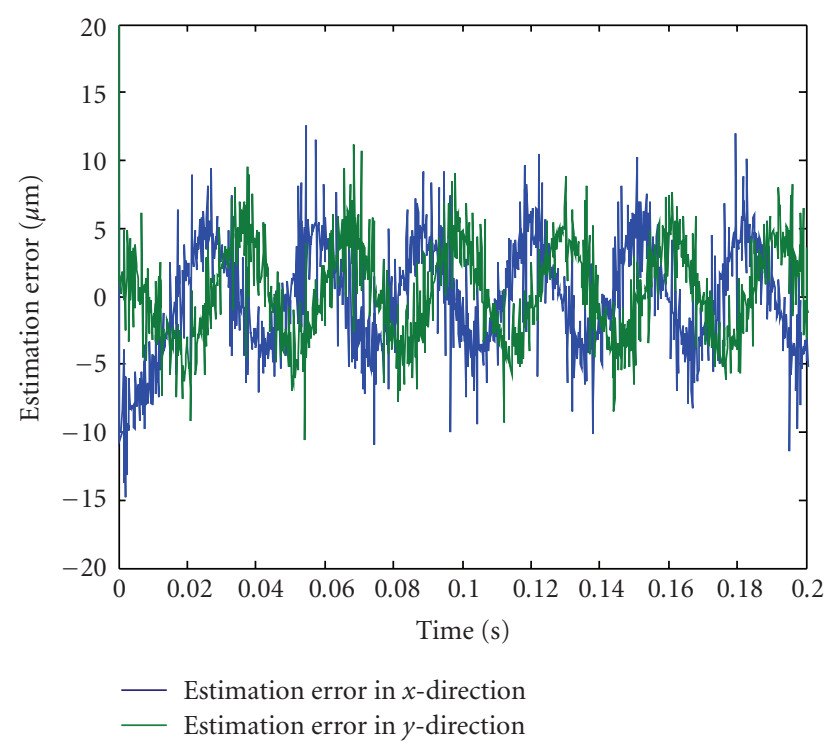

Figure 5: The estimation error of the observer in $x$-and $y$-directions.

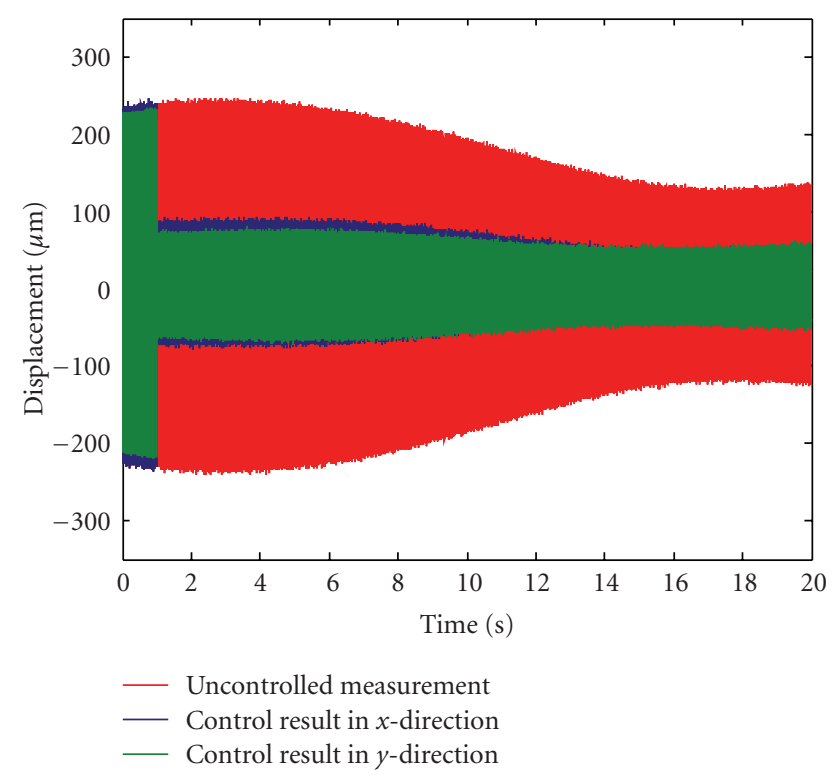

FIgUre 6: Rotor displacement with the CAFAC model when control is switched on after 3 seconds.

model has not yet been successfully used in the real environment, whereas the PEM identification has been proven to work and to provide a reduction of the vibrations up to $90 \%$ depending on how the controller is tuned.

\section{Conclusions}

In this paper, we used a knowledge-based Artificial FishSwarm optimization algorithm to identify the parameters of an actuator model in an electrical machine. The culture framework was invested to direct the crossover operation in the AFA. In the culture framework, the situation knowledge

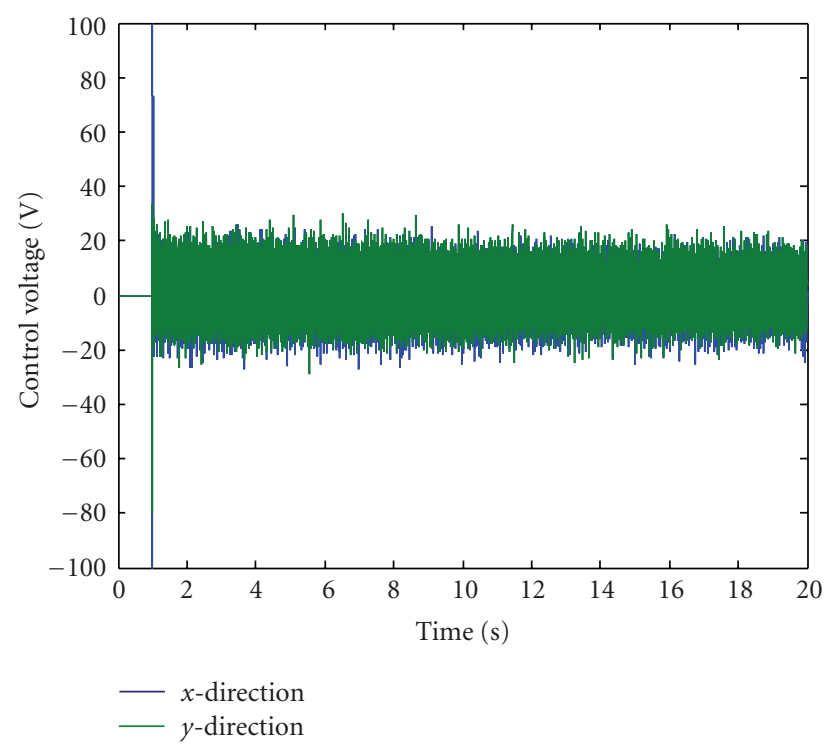

FIGURE 7: Control voltage of the LQ controller with CAFAC model when control is switched off after 3 seconds.

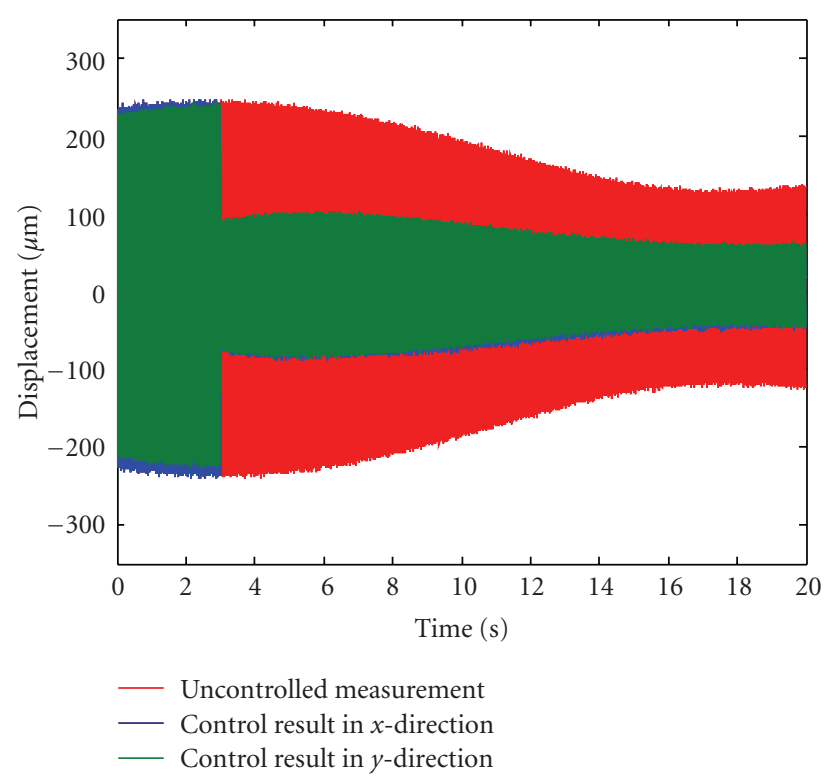

FIGURE 8: Rotor displacement with the PEM model when control is switched on after 3 seconds.

and the normative knowledge were employed to guide the evolution of the Artificial Fish-Swarm optimization. The crossover operation can help the artificial fish jump out of the local optimum without losing the characteristics of the previous generation. The proposed knowledge-based Artificial Fish-Swarm optimization can improve the performance of the original Artificial Fish-Swarm optimization and can be applied, for example, to find parameter values for a model of an actuator used for vibration control of rotor in an induction motor. Realistic values for the components of a structural first-principles electromechanical model were obtained, which improved the earlier identification results 


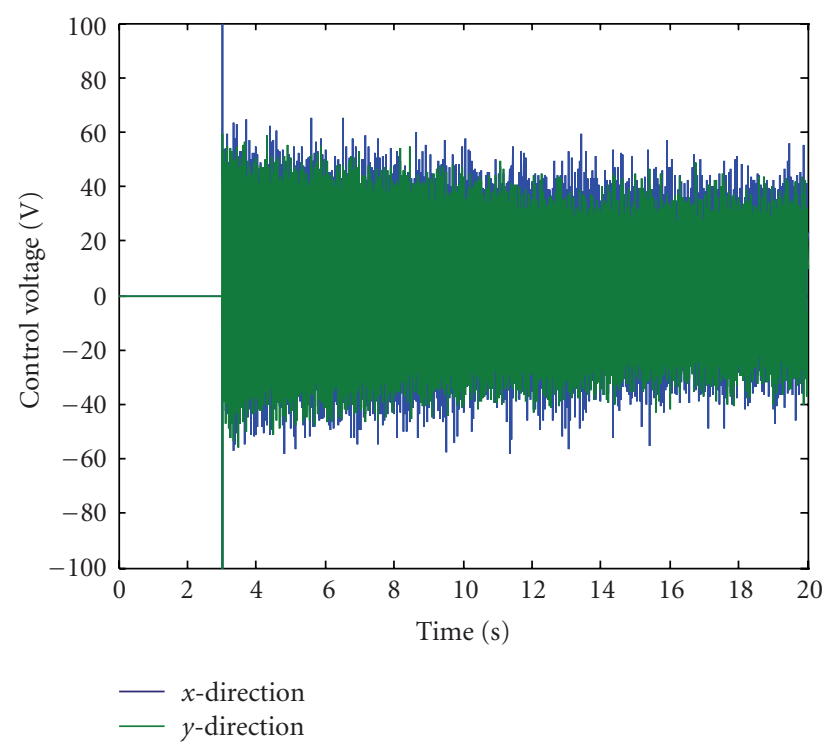

FIGURE 9: Control voltage of the LQ controller with PEM model when control is switched on after 3 seconds.

considerably. The results compared well with those obtained by PEM identification, but because the latter does not provide physical parameter values, the method presented in the paper can be considered an improvement. Also, the natureinspired evolutionary algorithms considered are particularly interesting, because they are computationally effective and reasonably easy to understand. Also, they do not need computation of gradients. For the future works, a timevarying model must be considered. In order to do that, more knowledge in the culture framework should be invested and more swarm intelligence must be tested to succeed in parameter identification.

\section{Acknowledgment}

This research work was funded by the Academy of Finland under Grants 135225 and 127299 and the NSFC under Grant no. 60874084 .

\section{References}

[1] R. K. Ursem, Models for evolutionary algorithms and their applications in system identification and control optimization, Ph.D. dissertation., Department of Computer Science University of Aarhus, Aarhus University, Aarhus, Denmark, 2003.

[2] Y. H. Shi and R. Eberhart, "Parameter selection in particle swarm optimization," in Proceedings of the 7th Annual Conference on Evolutionary Programming, pp. 591-600, Washington, DC, USA, 1998.

[3] T. Kumon, M. Iwasaki, T. Suzuki, T. Hashiyama, N. Matsui, and S. Okuma, "Nonlinear system identification using genetic algorithm," in Proceedings of the 26th Annual Conference of the IEEE Electronics Society (IECON '00), vol. 4, pp. 2485-2491, 2000.
[4] R. Storn, "System design by constraint adaptation and differential evolution," IEEE Transactions on Evolutionary Computation, vol. 3, no. 1, pp. 22-34, 1999.

[5] E. Bonabeau, M. Dorigo, and G. Theraulaz, Swarm Intelligence: From Natural to Artificial Systems, Oxford University Press, New York, NY, USA, 1999.

[6] X. L. Li, Z. J. Shao, and JI. X. Qian, "Optimizing method based on autonomous animats: fish-swarm algorithm," System Engineering Theory and Practice, vol. 22, no. 11, p. 32, 2002.

[7] R. Eberhart and J. Kennedy, "A new optimizer using particle swarm theory," in Proceedings of the 6th International Symposium on Micro Machine and Human Science, pp. 39-43, Nagoya, Japan, 2002.

[8] Y. Shi and R. Eberhart, "A modified particl swarm optimizer," in Proceedings of the IEEE World Congress on Computational Intelligence, pp. 69-73, Anchorage, Alaska, USA, 1998.

[9] U. Baumgartner, C. H. Magele, and W. Renhart, "Pareto optimality and particle swarm optimization," IEEE Transactions on Magnetics, vol. 40, no. 2, pp. 1172-1175, 2004.

[10] M. S. Voss and X. Feng, "Emergent system identification using particle swarm optimization," in The International Society for Optical Engineering, vol. 4512 of Proceedings of SPIE, Hutchinson Island, Fla, USA, June 2001.

[11] S. Z. Rizvi and H. N. Al-Duwaish, "A PSO-Subspace algorithm for identification of Hammerstein models," in Proceedings of the IFAC Conference on Control Applications of Optimisation, p. 5, Agora, Finland, 2009.

[12] V. Katari, S. Malireddi, S. K. S.bendapudi, and G. Panda, "Adaptive nonlinear system identification using comprehensive learning PSO," in Proceedings of the 3rd International Symposium on Communications, Control, and Signal Processing (ISCCSP '08), pp. 434-439, Las Vegas, Nev, USA, 2008.

[13] S. Chen, T. Mei, M. Luo, and X. Yang, "Identification of nonlinear system based on a new hybrid gradient-based PSO algorithm," in Proceedings of the International Conference on Information Acquisition (ICIA '07), pp. 265-268, Jeju City, Korea, July 2007.

[14] Y. Liu and X. He, "Modeling identification of power plant thermal process based on PSO algorithm," in Proceedings of the American Control Conference (ACC '5), pp. 4484-4489, Portland, Ore, USA, June 2005.

[15] E. Král, V. Lubomír, V. Pollinay, and P. Varacha, "Usage of PSO algorithm for parameters identification of district heating," Latest Trends on Systems, vol. 2, no. 657, p. 659, 2010.

[16] Y.-L. Zhou, Q.-Y. He, and M.-F. Xu, "Application of artificial fish-swarm algorithm in gas-solid fluidized bed flow regime identification," Chemical Engineering, vol. 38, no. 7, pp. 3942+50, 2010 .

[17] R. G. Reynolds, "An introduction to cultural algorithms," in Proceedings of the 3rd Annual Conference on Evolutionary Programming, pp. 131-139, San Diego, Calif, USA, 1994.

[18] R. G. Reynolds and B. Peng, "Cultural algorithms: modeling of how cultures learn to solve problems," in Proceedingsof the 16th IEEE International Conference on Tools with Artificial Intelligence (ICTAI '04), pp. 166-172, Boca Raton, Fla, USA, November 2004.

[19] C. J. Chung, Knowledge-based approaches to self-adaptation in cultural algorithms, Ph.D. Dissertation, Department of Computer Science, Wanyne State University, Detroit, Mich, USA, 1997.

[20] R. G. Reynolds and C. J. Chung, "Knowledge-based self-adaptation in evolutionary programming using cultural algorithms," in Proceedings of the IEEE Conference on Evolutionary 
Computation (ICEC 97), pp. 71-75, Indianapolis, Ind, USA, 1997.

[21] Robert G. Reynolds and Chung, "Knowledge-based self-adaptation in evolutionary programming using cultural algorithms," in Proceedings of the IEEE Conference on Evolutionary Computation (ICEC '97), pp. 71-75, Indianapolis, Ind, USA, 1997.

[22] L. S. Dos Coelho and V. C. Mariani, "An efficient particle swarm optimization approach based on cultural algorithm applied to mechanical design," in Proceedings of the IEEE Congress on Evolutionary Computation (CEC '06), pp. 10991104, Vancouver, Canada, July 2006.

[23] Y. Huang, Y. Xu, and G. Chen, "The culture-based particle swarm optimization algorithm," in Proceedings of the 4th International Conference on Natural Computation (ICNC '08), pp. 256-262, Jinan, China, October 2008.

[24] Y. Wu, X.-Z. Gao, X.-L. Huang, and K. Zenger, "A cultural Particle Swarm Optimization algorithm," in Proceedings of the 2010 6th International Conference on Natural Computation (ICNC '10), vol. 5, pp. 2505-2509, Yantai, China, 2010.

[25] Y. Wu, X.-Z. Gao, X.-L. Huang, and K. Zenger, "A hybrid optimization method of particle swarm optimization and cultural algorithm," in Proceedings of the 6th International Conference on Natural Computation (ICNC '10), vol. 5, pp. 2515-2519, Yantai, China, 2010.

[26] X. Z. Gao, Y. Wu, K. Zenger, and X. Huang, "A knowledgebased artificial fish-swarm algorithm," in Proceedings of the 13th IEEE International Conference on Computational Science and Engineering (CSE '10), pp. 327-332, Hong Kang, China, 2010.

[27] L. D. S. Coelho, R. C. T. Souza, and V. C. Mariani, "Improved differential evolution approach based on cultural algorithm and diversity measure applied to solve economic load dispatch problems," Mathematics and Computers in Simulation, vol. 79, no. 10, pp. 3136-3147, 2009.

[28] A. Sinervo, Modeling and control of flexural rotor vibration of a two-pole cage induction motor, M.S. thesis, Department of electrical Engineering, Faculty of electronics, Communications and Automation, Helsinki University of Technology, Espoo, Finland, 2008.

[29] A. Laiho, A. Sinervo, J. Orivuori, K. Tammi, A. Arkkio, and K. Zenger, "Attenuation of harmonic rotor vibration in a cage rotor induction machine by a self-bearing force actuator," IEEE Transactions on Magnetics, vol. 45, no. 12, Article ID 5326448, pp. 5388-5398, 2009.

[30] J. Orivuori, K. Zenger, and A. Sinervo, "Active control of rotor vibrations by advanced control methods," in Proceedings of the 16th International Congress on Sound and Vibration-Recent Developments in Acoustics, Noice and Vibration, M. Pawelczyk and D. Bismor, Eds., Krakow, Poland, 2010.

[31] A. Laiho, K. Tammi, and V. Vidquist, "Modelling of flexural rotor vibration and time-periodic system dynamics in a twopole cage induction machine equipped with a self-bearing force actuator," in Proceedings of the IFAC Workshop on Periodic Systems, Bogazici University, Turkey, 2010.

[32] G. Genta, Vibration of Structures and Machines: Practical Aspects. Section 6.4., Springer, New York, NY, USA, 1999.

[33] A. Laiho, K. Tammi, K. Zenger, and A. Arkkio, "A model-based flexural rotor vibration control in cage induction electrical machines by a built-in force actuator," Electrical Engineering, vol. 90, no. 6, pp. 407-421, 2008.

[34] T. P. Holopainen, A. Tenhunen, E. Lantto, and A. Arkkio, "Unbalanced magnetic pull induced by arbitrary eccentric motion of cage rotor in transient operation. Part 1: analytical model," Electrical Engineering, vol. 88, no. 1, pp. 13-24, 2005.

[35] X. P. Wang and L. M. Cao, Genetic Algorithm: Theory, Application and Software Implementation, Xi'an JiaoTong University Press, Xi'an, China, 2002.

[36] P. Krause, Analysis of Electric Machinery, McGraw-Hill, New York, NY, USA, 1987. 

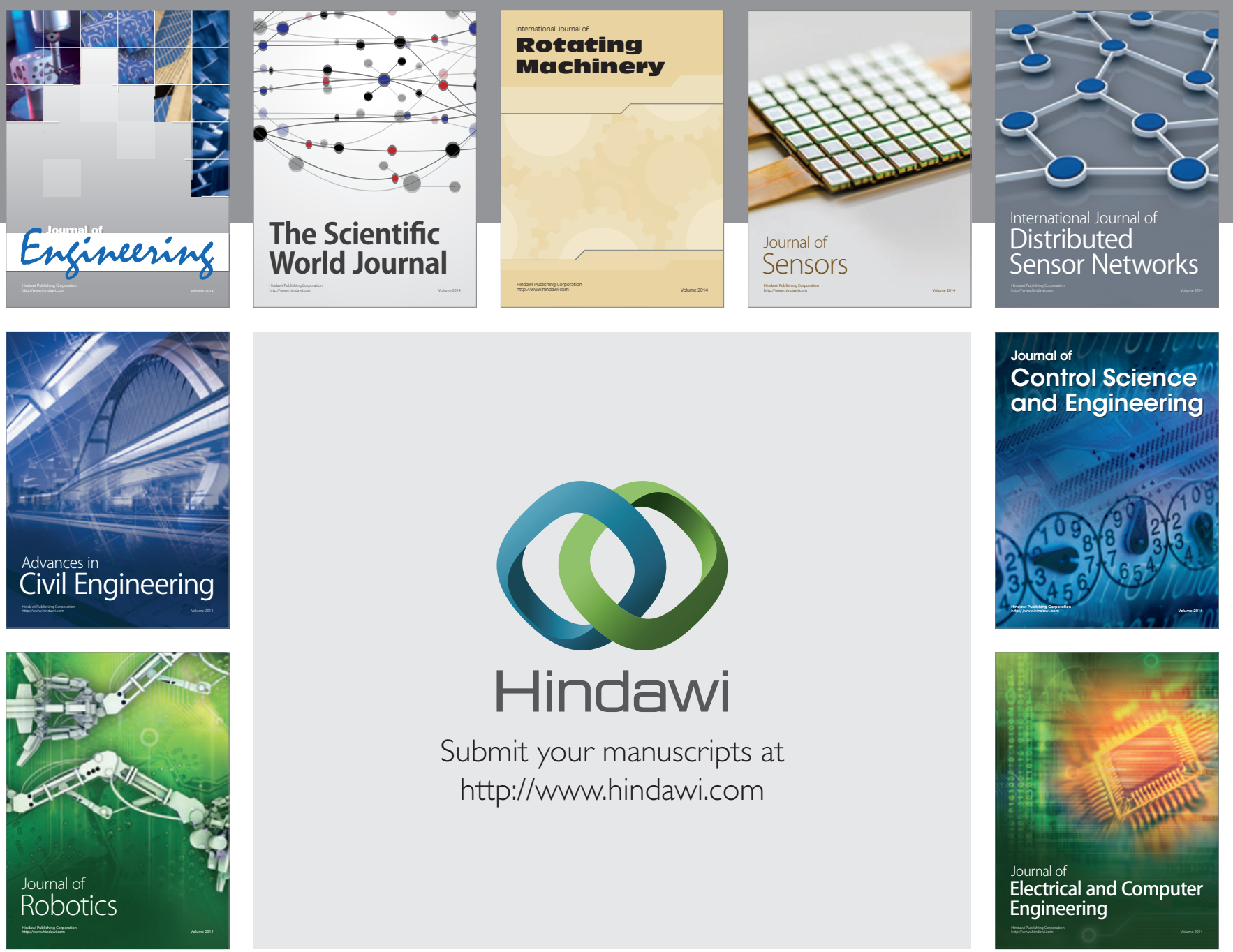

Submit your manuscripts at

http://www.hindawi.com
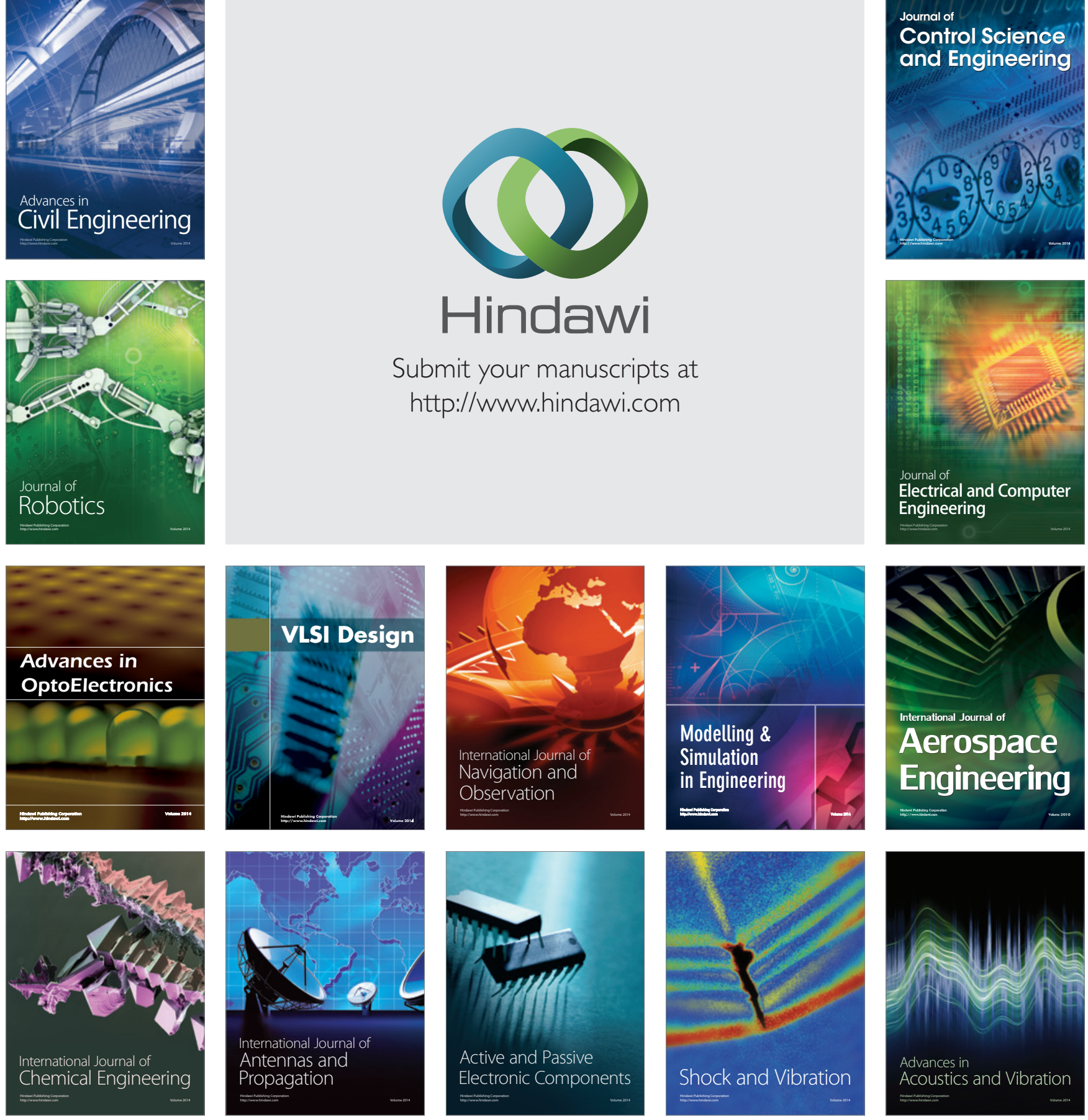